\title{
Lab Result CM Domain
}

National Cancer Institute

\section{Source}

National Cancer Institute. Lab Result CM Domain. NCI Thesaurus. Code C154630.

This table is used to store quantitative and qualitative measurements from blood and other body specimens. 